Abschließend möge an Hand eines numerischen Beispiels das magnetische Verhalten von zwei Halbleitern verglichen werden, von denen der eine doppelt ionisierbare Störstellen der oben ausführlich diskutierten Art enthalte (Fall 1), der andere jedoch zwei Störstellenarten einfacher Ionisierbarkeit (Fall 2). Bei gleicher Dichte aller Störstellen und paarweise gleichen Umladungsenergien werden die Trägerzahlen (und damit auch wesentlich die elektrischen Eigenschaften) in beiden Fällen (nahezu) gleich sein. Merkliche Unterschiede zeigt dagegen das magnetische Verhalten dieser Störstellen. Im Fall 1 gilt Formel (7); im Fall 2 tritt an ihre Stelle

$$
\varkappa=\frac{\mu_{\mathrm{B}}^{2}}{k T}\left(\frac{N_{1}}{1+\frac{1}{2} \exp \left(\frac{E^{\prime}-\zeta}{k T}\right)}+\frac{N_{2}}{1+\frac{1}{2} \exp \left(\frac{E^{\prime \prime}-\zeta}{k T}\right)}\right) .
$$

Für $T=100{ }^{\circ} \mathrm{K}$ und $300{ }^{\circ} \mathrm{K}, N_{1}=N_{2}=N_{\mathrm{d}}=10^{1 \tau}$ $\mathrm{cm}^{-3}, E^{\prime}=-0,03 \mathrm{eV}, E^{\prime \prime}=-0,09 \mathrm{eV}$ gibt Abb. 1 den Verlauf von $\varkappa(\zeta)$ wieder. Die im Diagramm zugelassene Möglichkeit, $\zeta$ (durch zusätzliche Dotierung, die hier nicht diskutiert wird) willkürlich zu verschieben, bleibe außer Betracht. Dann gehört zu

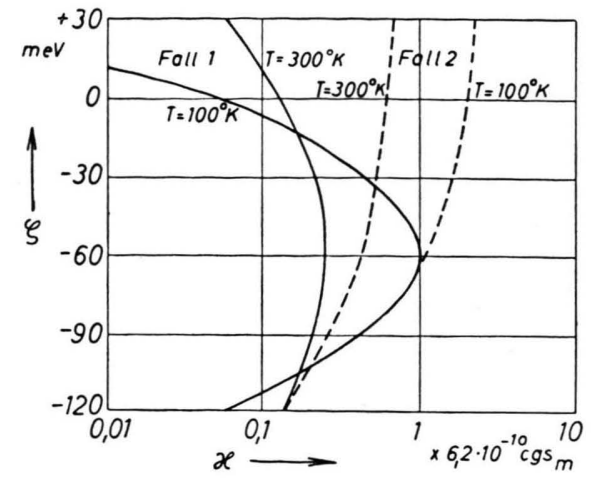

Abb. 1. Verlauf der Störstellensuszeptibilität für einen doppelt ionisierbaren Donator (Fall 1) oder zwei einfach ionisierbare Donatoren derselben Umladungsenergien (Fall 2).

niederen Temperaturen ein $\zeta$-Wert über $-30 \mathrm{meV}$, zu hohen Temperaturen ein $\zeta$-Wert unter $-90 \mathrm{meV}$. Das bedeutet, daß mit steigender Temperatur im Fall 1 von kleinen Suszeptibilitätswerten kommend ein Maximum durchlaufen wird, dagegen im Fall 2 die Suszeptibilität mit großen Werten beginnend monoton abnimmt.

\title{
Über Stickstoffionen im Plasma
}

\author{
Von H. Dreeskamp * \\ Aus dem Institut für Physikalische Chemie der Universität Bonn \\ (Z. Naturforschg. 12 a, 876-881 [1957]; eingegangen am 5. August 1957)
}

\begin{abstract}
Mit einem doppelfokussierenden Massenspektrometer wurden die aus dem Plasma einer StickstoffNiederdruck-Entladung effundierenden Ionen gemessen. Es traten nur $\mathrm{N}^{+}$- und $\mathrm{N}_{2}{ }^{+}$-Ionen auf. Ihr Verhältnis wurde in Abhängigkeit von Druck und Stromstärke in der Entladung bestimmt. Ferner wurden die Stickstoff-Ionen in einem Driftraum in Abhängigkeit von $\mathbb{E} / P$ massenspektrometrisch untersucht. Neben $\mathrm{N}^{+}$- und $\mathrm{N}_{2}^{+}$- traten $\mathrm{N}_{3}{ }^{+}$-Ionen auf. Die Ergebnisse lasen sich durch ein von $\mathfrak{E} / P$ abhängiges Gleichgewicht $\mathrm{N}^{+}+\mathrm{N}_{2} \rightleftharpoons \mathrm{N}_{3}{ }^{+}$erklären. Durch eine rechnerische Bestimmung des Radialfeldes vor der Wand des Entladungsgefäßes wird gezeigt, daß unter den Bedingungen der vorliegenden Arbeit $\mathrm{N}_{3}{ }^{+}$-Ionen nicht unter den aus dem Plasma einer Entladung effundierenden Ionen beobachtet werden können, während mit ihrer Existenz im Zentrum der Entladung gerechnet werden muß.
\end{abstract}

\section{Problemstellung}

Stickstoffionen im Kathodenfallgebiet einer Gasentladung sind von TüxeN ${ }^{1}$ nach der Kanalstrahlmethode mit einem Parabelspekrographen untersucht worden. $\mathrm{Er}$ fand $\mathrm{N}^{+}$- und $\mathrm{N}_{2}{ }^{+}$-Ionen, aber weder negative noch Anlagerungs-Ionen. Mit einem rich-

* z. Zt. Notre Dame University, Department of Chemistry, Indiana, U.S.A.

1 O. Tüxen, Z. Phys. 103, 463 [1935]. tungsfokussierenden Massenspektrometer untersuchte LuHR ${ }^{2,3}$ Stickstoffionen, die aus einer Gasentladung stammten und in einer Driftstrecke Um- oder Anlagerungen erlitten hatten. $\mathrm{Er}$ fand $\mathrm{N}^{+}$- und $\mathrm{N}_{2}^{+}$-Ionen und bei Drucken über 0,2 Torr auch $\mathrm{N}_{3}^{+}$- und $\mathrm{N}_{4}^{+}$Ionen. In einer neueren Arbeit von $\mathrm{V}_{\text {ARNEY }}{ }^{4}$ über die Driftgeschwindigkeit von Ionen wird die Reak-

2 O. Luhr, Phys. Rev. 38, 1736 [1931].

3 O. Luhr, Phys. Rev. 44, 459 [1933].

${ }^{4}$ R. N. Varney, Phys. Rev. 89, 708 [1953]. 


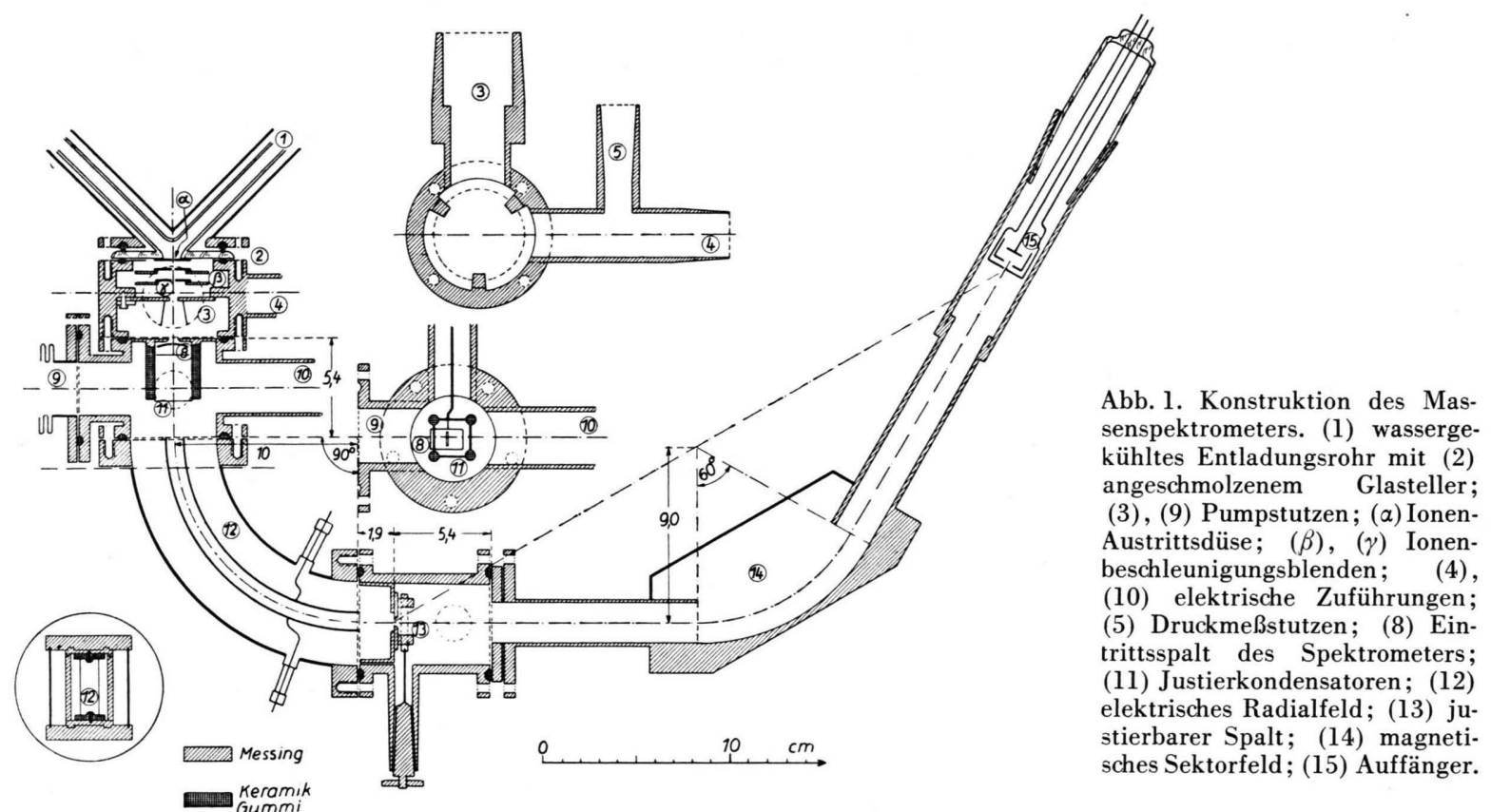

tion $\mathrm{N}_{2}{ }^{+}+\mathrm{N}_{2} \rightarrow \mathrm{N}_{4}{ }^{+}$im Gebiet $[\& / P<100 \mathrm{~V} / \mathrm{cm}$ Torr angenommen, um Anomalien der Driftgeschwindigkeit zu erklären.

Ziel dieser Arbeit war es, die Ionen im Plasma der positiven Säule selbst zu untersuchen und die Ergebnisse älterer Arbeiten zu überprüfen.

\section{Stickstoffionen im Plasma}

\section{Experimentelles}

Da bei den vorliegenden Untersuchungen ein relativ kleines Auflösungsvermögen des Massenspektrometers (M.S.) von $M / \Delta M \sim 50$ genügte, die durch die Gasentladung bedingte Energiebreite der Ionen aber groß war (einige Volt), ergab sich der kleinste experimentelle Aufwand bei Verwendung eines kleinen doppelfokussierenden M.S., dessen Konstruktion in Abb. 1 dargestellt ist. Würde - wie in älteren Arbeiten ${ }^{5,6,7}$ eine metallische Ziehblende als Grenze zwischen dem Plasma und dem M.S. verwendet werden, so träten neben Rekombinationen an der Metallfläche die schlecht zu übersehenden Raumladungen im Dunkelraum vor der Ziehblende auf. Es war also zu fordern: 1. Die Metallfläche der Düse zwischen dem Entladungsplasma und dem M.S. sei so klein wie möglich. 2. Der Gesamtstrom auf diese Düse sei wie bei einer isolierenden Glaswand gleich Null. Da somit Ladungsträger allein durch die Kräfte im Plasma durch die Düse getrieben

5 R. Boyd, Nature, Lond. 165, 142 [1950].

6 J. Spencer-Smith, Phil. Mag. 19, 1016 [1935].

7 R. W. Lunt u. A. H. GREGG, Trans. Faraday Soc. 234, 1063 [1940]. werden, sei dieser Mechanismus nach einem Vorschlag von PAHL "ambipolare Effusion der Ladungsträger“" genannt. In einer früheren Arbeit ${ }^{8}$ ist die Herstellung der Düse a sowie der Aufbau des Vakuumsystems und die zum M.S. gehörige elektrische Schaltung angegeben. Die Speisung der Entladung erfolgte jedoch in der vorliegenden Arbeit aus einer stabilisierten Gleichstromquelle. Ferner wurde ein empfindlicherer Gleichstromverstärker zum Ionennachweis mit Registrierung durch einen Schreiber und eine Vorrichtung zur zeitproportionalen Änderung der elektrischen und magnetischen $\mathrm{Ab}$ lenkfkelder benutzt.

Mit dem M.S. wurde ein Auflösungsvermögen von $M / \Delta M=60$ bei einer Beschleunigungsspannung des M.S. von $400 \mathrm{~V}$ und einer mittleren Energiebreite der Ionen von $\Delta U=6 \mathrm{~V}$ erreicht.

Während die absolute Ionenstromstärke auf den Auffänger des M.S. stark von den Betriebsdaten abhing (vornehmlich Druck und Justierung des M.S.), interessierten im Rahmen dieser Untersuchungen allein Aussagen über das Verhältnis der Ströme der einzelnen Ionensorten, das in einer weiter unten zu bestimmenden Weise vom Verhältnis der stationären Ionenkonzentrationen im Plasma abhängig ist. Es wurde sorgfältig untersucht, ob dieses Verhältnis vom Betrieb des M.S. abhängig war, vor allem vom Strom auf die Düse ( $\alpha$, Abb. 1) - dieser Strom war maximal $3 \cdot 10^{-6} \mathrm{~A}$ oder $1 / 10$ des Sondensättigungsstromes auf die Düse - und den Fokussierungseigenschaften des M.S. Es ergab sich eine Konstanz von $4 \%$ und eine Reproduzierbarkeit von $8 \%$.

8 H. D. Beckey u. H. Dreeskamp, Z. Naturforschg. 9 a, 735 [1954]. 


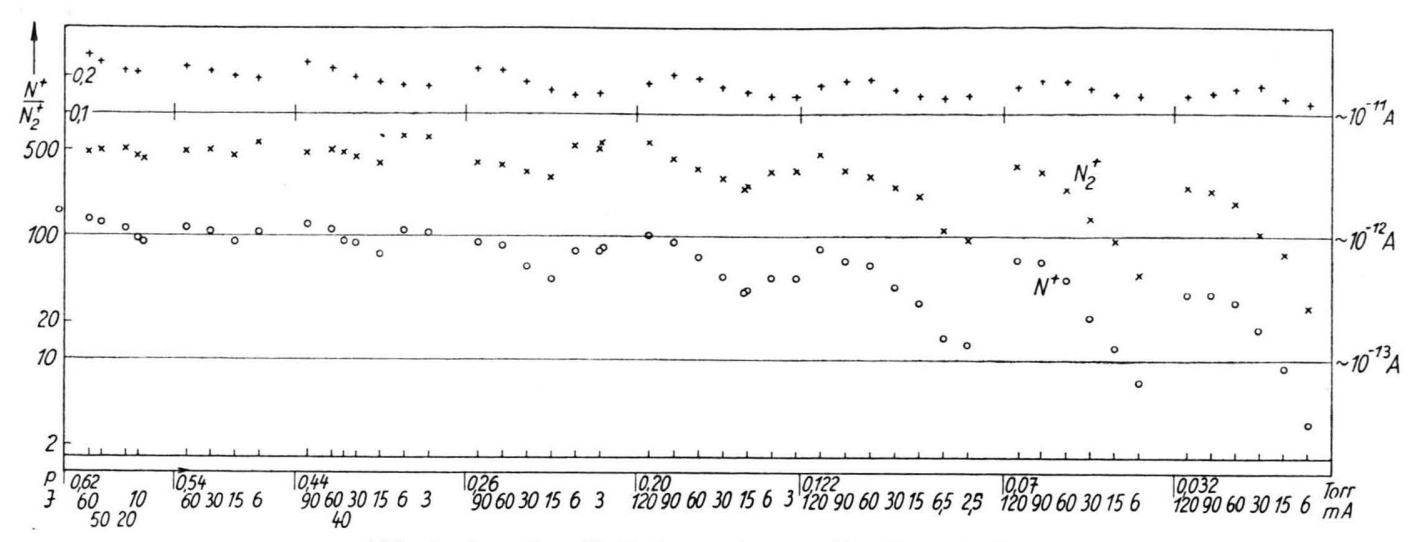

Abb. 2. Aus dem Entladungsplasma effundierende Ionen.

\section{Meßergebnisse}

Neben einem Peak bei der Masse 40, der durch die Verunreinigung des verwendeten. Stickstoffs mit $0,3 \% \mathrm{Ar}$ hervorgerufen wurde, traten allein $\mathrm{N}^{+}$- und $\mathrm{N}_{2}{ }^{+}$-Ionen auf. In dem mit dieser Apparatur zugänglichen Gebiet: Entladungsstromstärke $3 \leqq I \leqq 120$ $\mathrm{mA}$, Druck in der Entladung $0,03 \leqq P \leqq 0,8$ Torr, war die Intensität anderer Ionen - also etwa $\mathrm{N}_{3}{ }^{+}$ oder $\mathrm{N}_{4}^{+}$- kleiner als $0,02 \%$ des Gesamtionenstromes.

In Abb. 2 sind die Ströme der Ionen $\mathrm{N}^{+}$und $\mathrm{N}_{2}^{+}$ auf den Auffänger des M.S. sowie ihre Verhältnisse in Abhängigkeit von den Daten der Entladung wiedergegeben. Das Verhältnis $j\left(\mathrm{~N}^{+}\right) / j\left(\mathrm{~N}_{2}^{+}\right)$liegt zwischen 0,3 und 0,1 und steigt im allgemeinen mit wachsendem Druck und wachsender Stromstärke, doch läßt sich eine einfache analytische Beziehung zwischen $j\left(\mathrm{~N}^{+}\right) / j\left(\mathrm{~N}_{2}{ }^{+}\right), I$ und $P$ kaum aufstellen. Der Zusammenhang der gemessenen Größen mit den Ionenkonzentrationen im Entladungsplasma wird später behandelt.

\section{Stickstoffionen im Driftraum}

Da unter den aus einer Stickstoffentladung effundierenden Ionen keine $\mathrm{N}_{3}{ }^{+}$oder $\mathrm{N}_{4}{ }^{+}$gefunden wurden, stellte sich die Frage, ob sich diese Ionen durch Anlagerung von $\mathrm{N}^{+}$oder $\mathrm{N}_{2}^{+}$an $\mathrm{N}_{2}$ bilden, wenn diese Ionen unter dem Einfluß eines schwachen Feldes durch Stickstoff bewegt werden.

Um diese Frage zu klären, wurde die oben beschriebene Apparatur in der folgenden Weise geändert: Zwischen das Entladungsrohr und das M.S. wurde ein Flanschstück (Abb. 3) montiert, die Düse vom Teller des Entladungsrohres entfernt und statt dessen ein Glas-Metall-Kästchen (Driftraum) aufgekittet. Dem
Entladungsrohr zugewandt befand sich ein Drahtgitter, durch das Ionen aus der Entladung in den Driftraum diffundierten. Durch eine zwischen dieses Gitter und den Boden des Driftraumes angelegte Spannung $Y$ wurden Ionen auf das M.S. zu beschleunigt, erlitten bei Stößen Umladungen und Anlagerungen und traten durch ein Loch im Boden des Driftraumes in das M.S. ein, das die in Abb. 1 angegebene Form hatte.

Bei relativ hohen Drucken $(P>0,2$ Torr $)$ traten nur Ionen auf, deren Energie der durchlaufenen Beschleunigungsspanung des M.S. entsprach, während bei niedrigeren Drucken neben diesen Ionen auch solche auftraten, die eine bis zum Betrage der an den Elektroden des Driftraumes liegenden Spannung größere Energie hatten. Bei diesen Drucken war of-

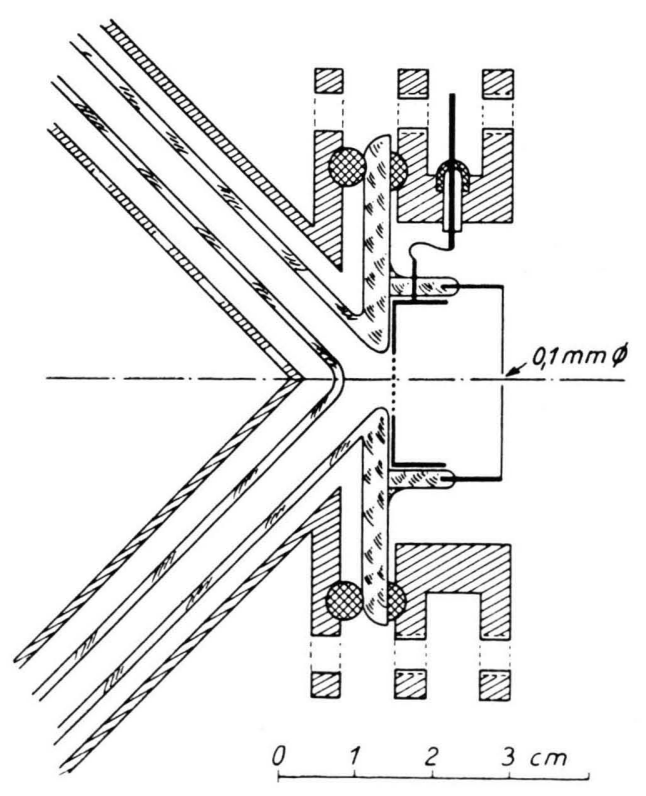

Abb. 3. Konstruktion des Driftraumes. 
fensichtlich die Bedingung: Freie Weglänge $\lambda$ der Ionen $\ll$ Driftstrecke $d$ nicht mehr hinreichend erfüllt, so daß Messungen nur bei $P>0,2$ Torr gemacht wurden.

Das häufigste Ion war wieder $\mathrm{N}_{2}^{+}$. Daneben traten, abhängig von der relativen Feldstärke $\mathbb{E} / P$ im Driftraum, die Ionen $\mathrm{N}^{+}$und $\mathrm{N}_{3}^{+}$auf, während $\mathrm{N}_{4}^{+}$-Ionen nicht gefunden wurden (Konzentration $<0,5 \%$ des gesamten Ionenstromes).

Wie die Abb. 4 zeigt, lassen sich die Ionenströme (in Prozenten des integralen Ionenstromes) als Funktion der relativen Feldstärke im Driftraum darstellen. Die Stromstärke der Entladung war hierbei $I=80 \mathrm{~mA}$. Die Reproduzierbarkeit betrug bei diesen Messungen $\pm 10 \%$ des Meßwertes.

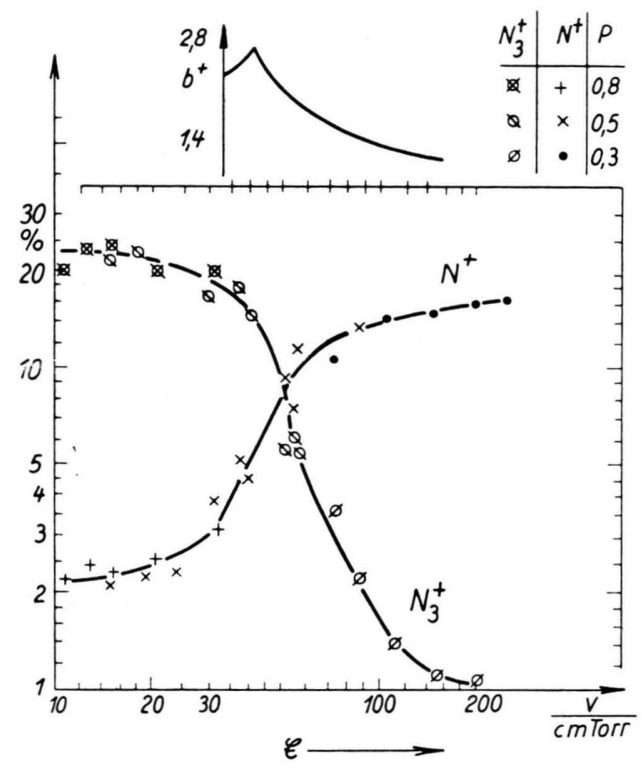

Abb. 4. Aus dem Driftraum effundierende Ionen.

\section{Diskussion der Ergebnisse}

Zunächst seien die Messungen der Stickstoffionen im Driftraum diskutiert: Wie Abb. 4 zeigt, ist die Summe der Ströme $j\left(\mathrm{~N}^{+}\right)$und $j\left(\mathrm{~N}_{3}{ }^{+}\right)$ungefähr konstant. Außerdem ist das Verhältnis

$$
\frac{j\left(\mathrm{~N}^{+}\right)+j\left(\mathrm{~N}_{3}^{+}\right)}{j\left(\mathrm{~N}_{2}{ }^{+}\right)}=0,26 \quad(\text { bei } P=0,5 \text { Torr })
$$

etwa gleich dem Verhältnis der direkt aus dem Plasma austretenden Atom- und Molekülionen (Abb. 2) bei gleichen Werten von $I$ und $P$.
Nimmt man an, daß die beiden Ionensorten $\mathrm{N}^{+}$ und $\mathrm{N}_{2}^{+}$auch in diesem Verhältnis aus dem Plasma durch das Drahtgitter in den Driftraum treten - wegen einer Störung der Entladung durch das Metall des Gitters ist diese Voraussetzung nicht unbedingt erfüllt - , so folgt:

1. $\mathrm{N}_{3}{ }^{+}$-Ionen werden nur bei kleinen Werten der relativen Feldstärke gebildet; bei höheren Werten werden sie nicht gebildet oder sind instabil.

2. Die einfachste Annahme zur Bildung der $\mathrm{N}_{3}{ }^{+}$. Ionen ist:

$$
\mathrm{N}^{+}+\mathrm{N}_{2}+\mathrm{N}_{2} \rightarrow \mathrm{N}_{3}^{+}+\mathrm{N}_{2} .
$$

3. $\mathrm{N}_{2}{ }^{+}$-Ionen passieren den Driftraum ungeändert, von Umladungen abgesehen.

Die 3. Folgerung ist nur dann richtig, wenn die Entladung durch das Drahtgitter des Driftraumes nicht gestört wird und die im ersten Teil dieser Arbeit gemessenen Ionenkonzentrationen (Abb. 2) in den Driftraum eintreten. Da es im Rahmen dieser Arbeit nicht möglich war, gleichzeitig die Zusammensetzung der in den Driftraum ein- und austretenden Ionen zu messen, ist auch eine Umwandlung von $\mathrm{N}_{2}{ }^{+}$ in $\mathrm{N}^{+}$und damit in $\mathrm{N}_{3}{ }^{+}$nicht auszuschließen.

In Abb. 4 ist ferner die von VARNeY ${ }^{4}$ experimentell bestimmte Kurve der Ionenbeweglichkeit $b^{+}$für Stickstoff eingetragen. Um die ungewöhnliche Änderung von $b^{+}$mit $\& / P$ zu erklären, nimmt VARNEy den Prozeß: $\mathrm{N}_{2}{ }^{+}+\mathrm{N}_{2} \rightarrow \mathrm{N}_{4}{ }^{+}$an, indem er sich auf den Nachweis von $\mathrm{N}_{4}^{+}$-Ionen durch LuHR ${ }^{2,3}$ stützt. Da die Existenz von $\mathrm{N}_{4}{ }^{+}$-Ionen in dieser Arbeit nicht bestätigt werden konnte, die Änderung von $b^{+}$mit $\mathbb{E} / P$ aber bei dem gleichen Wert erfolgt wie die Umwandlung von $\mathrm{N}^{+}$in $\mathrm{N}_{3}{ }^{+}$, ist dieser Prozeß die wahrscheinliche Ursache für die experimentell gefundene $\mathrm{Ab}$ hängigkeit von $b^{+}$mit $\mathbb{E} / P$.

Um die Messungen der aus dem Plasma effundierenden Ionen zu diskutieren, wurde die Größe der relativen Feldstärke in der hier vorliegenden Arbeit vor der Wand des Entladungsgefäßes berechnet, in einer Zone also, die die Ionen auf ihrem Weg aus dem Plasma kurz vor dem Eintritt in das M.S. zu passieren hatten.

Die Sснотткysche Theorie der positiven Säule liefert für den Verlauf der radialen Feldstärke den Ausdruck (siehe z. B. Anm. ${ }^{9}$ )

9 A.v. Engel u. M. Steenbeck, Elektrische Gasentladungen II, J. Springer, Berlin 1934, S. 90. 


$$
\mathfrak{F}_{\mathrm{r}}=\frac{2,4}{R} \frac{k T^{-}}{e} J_{1}\left(\frac{2,4}{R} r\right) \frac{n_{0}}{n}
$$

( $R=$ Radius des Entladungsrohres, $k=$ Boltzmann-Konstante, $e=$ Elementarladung, $T^{-}=$Elektronentemperatur, $J_{1}=$ BesseL-Funktion 1 . Ordnung, $r=$ radialer $\mathrm{Ab}$ stand von der Rohrachse, $n=$ Trägerkonzentration, $n_{0}=n$ für $\left.r=0\right)$.

Addiert man zu diesem Wert der Feldstärke noch den Wert der Längsfeldstärke $\mathfrak{F}_{\mathrm{L}}$, der experimentell leicht bestimmbar und den Angaben von SeELiger ${ }^{10}$ entnommen ist, so läßt sich die Feldstärke an der Rohrwand $E$ bestimmen, wenn $T^{-}$und $n_{0} / n$ bekannt sind.

$T^{-}$läßt sich nach der Sснотткyschen Theorie mit etwa $10 \%$ Genauigkeit berechnen. In dieser Arbeit sind die Angaben von Francis ${ }^{11}$ verwendet worden. Für das Ionisationspotential ist das des StickstoffMoleküls $\left(V_{\mathrm{I}}=15,5 \mathrm{eV}\right)$ angenommen und die geringe Konzentration der Atomionen vernachlässigt worden.

Aus der Gleichung für die Radialfeldstärke erkennt man, daß die Randbedingung $n_{\mathrm{R}}=0 \mathrm{zu}$ der Schwierigkeit unendlich großer Feldstärken in der Wandnähe führt. Von v. Engel und Steenbeck ist daher die Berechnung des Verhältnisses $n_{0} / n_{\mathrm{R}}$ aus dem Ansatz abgeleitet worden, daß der auf die Wand der Entladung treffende Ionenstrom in der Zeiteinheit dem im Plasma neugebildeten Ionenstrom gleich sein muß, während $n_{0}$ sich aus der Entladungsstromstärke berechnet. Dies führt auf die physikalisch sinnvolle Beziehung:

$$
n_{0} / n_{\mathrm{R}}=R / 1,66 \lambda,
$$

wobei $\lambda$ einen Mittelwert aus der freien Weglänge der Ionen und der Elektronen darstellt.

Dieser Ansatz zur Berechnung von $n_{0} / n_{\mathrm{R}}$ ist von FETZ $^{12}$ kritisiert worden, da er zu einer falschen Ionentemperatur in der Wandnähe führt. Durch eine experimentelle Bestimmung der Funktion $n=n(r)$ durch Yarnold und Holmes ${ }^{13}$ ist jedoch sichergestellt, da $\beta$ bei der Berechnung von $n_{0} / n_{\mathrm{R}}$ nach der obigen Gleichung kein allzu großer Fehler gemacht wird.

In Tab. 1 sind für $R=0,6 \mathrm{~cm}$ und $I=80 \mathrm{~mA}$ und für verschiedene Drucke die berechneten Werte aufgeführt.

Die Rechnung ergibt an der Wand des Entladungsrohres Werte der relativen Feldstärke, die so hoch

10 R. SEeliger, Physik der Gasentladungen, J. A. Barth, Leipzig 1934, S. 327.

\begin{tabular}{|c|c|c|c|c|c|c|}
\hline$P$ & Torr & 1 & 0,75 & 0,5 & 0,25 & 0,1 \\
\hline $\mathfrak{F}_{L}$ & $\frac{\mathrm{V}}{\mathrm{cm}}$ & 48 & 46 & 44 & 39 & 31 \\
\hline$\frac{E_{\mathrm{L}}}{P}$ & $\frac{\mathrm{V}}{\mathrm{cm} \cdot \text { Torr }}$ & 48 & 61,4 & 88 & 156 & 310 \\
\hline$T^{-}$ & $\cdot 1000^{\circ} \mathrm{K}$ & 18,5 & 20,2 & 25 & 29,5 & 37 \\
\hline$\frac{n_{0}}{n_{\mathrm{R}}}$ & & 226 & 189 & 113 & 56,5 & 22,6 \\
\hline $\mathfrak{E}_{\mathrm{R}}$ & $\frac{\mathrm{V}}{\mathrm{cm}}$ & 134 & 111 & 90 & 53,4 & 26,5 \\
\hline$E$ & $\frac{\mathrm{V}}{\mathrm{cm}}$ & 143 & 120 & 100 & 66 & 41 \\
\hline$\frac{E}{P}$ & $\frac{\mathrm{V}}{\mathrm{cm} \cdot \text { Torr }}$ & 143 & 160 & 200 & 264 & 410 \\
\hline
\end{tabular}

11 G. Francis, Handbuch der Physik XXII, 125 [1956].
Tab. 1.

sind, daß dort das Gleichgewicht $\mathrm{N}^{+}+\mathrm{N}_{2} \rightleftharpoons \mathrm{N}_{3}^{+}$ - wie in dieser Arbeit nachgewiesen wurde - vollständig auf der Seite der $\mathrm{N}^{+}$-Ionen liegt. Ionen, die aus dem M.S. durch die Düse effundieren, müssen aber auf der letzten Strecke ihres Weges diese Zone passieren.

In der Achse des Entladungsrohres ist $\mathcal{F}_{\mathrm{r}}=0$ und damit allein die Längsfeldstärke wirksam. Wie die Tabelle zeigt, wäre dort bei Drucken $P>0,75$ Torr eine Bildung von $\mathrm{N}_{3}{ }^{+}$-Ionen nicht ausgeschlossen.

Der Zusammenhang zwischen den stationären Ionenkonzentrationen $n_{\mathrm{i}}$ und $n_{\mathrm{k}}$ zweier Ionensorten $\mathrm{i}$ und $\mathrm{k}$ im Plasma und den im M.S. gemessenen Auffängerströmen $j_{\mathrm{i}}$ und $j_{\mathrm{k}}$ folgt aus dem Ausdruck für den Wandstrom einer Trägersorte ${ }^{14}$

$$
i_{\mathrm{w}}=-2 \pi R e D_{\mathrm{a}}\left(\frac{\mathrm{d} n}{\mathrm{~d} r}\right)_{r=R}=7,83 T^{-} k b^{+} n_{0}
$$

$\left(i_{\mathrm{w}}=\right.$ Stromdichte einer Trägersorte auf die Wand, $D_{\mathrm{a}}=$ ambipolarer Diffusionskoeffizient, $b^{+}=$Ionenbeweglichkeit).

Kann man die Umwandlung von Trägerarten ineinander und quadratische Prozesse ausschließen, so folgt aus:

$$
\frac{n_{\mathrm{i}}(r)}{n_{\mathrm{k}}(r)}=\frac{\mathrm{d} n_{\mathrm{i}}}{\mathrm{d} r}: \frac{\mathrm{d} n_{\mathrm{k}}}{\mathrm{d} r}=\text { const }
$$

unter der Voraussetzung, daß die Transmission des M.S. für alle Ionensorten gleich ist - diese Voraus-

12 H. Fetz, Ann. Phys., Lpz. 40, 579 [1941].

13 G. D. Yarnold u. S. Holmes, Phil. Mag. 22, 988 [1936].

14 Siehe Anm. ${ }^{11}$, S. 124. 
setzung ist bei einem Spektrometer der verwendeten Bauart hinreichend erfüllt - :

$$
\frac{j_{\mathrm{i}}}{j_{\mathrm{k}}}=\frac{b_{\mathrm{i}}^{+}}{b_{\mathrm{k}}{ }^{+}} \frac{n_{0 \mathrm{i}}}{n_{0 \mathrm{k}}}
$$

Beim Stickstoff sind nur die Beweglichkeiten für das Molekülion, nicht aber die für das Atomion bekannt und dürften auch für das Trägergas einer StickstoffEntladung schwer zu ermitteln sein. Da jedoch die Atomkonzentration in einer Stickstoff-Entladung nur etwa $1-2 \%$ beträgt ${ }^{15}$, ist damit zu rechnen, daß das Molekülion wegen der möglichen Umladungen eine kleinere Beweglichkeit besitzt, ähnlich wie in

15 W. Groth u. P. Warneck, Z. Phys. Chem. N. F. 11, 323 [1957].
Edelgasen das Atomion ${ }^{16}$. Der Einfluß quadratischer Prozesse dagegen ist nach einer Untersuchung von SPENKE $^{17}$ von vernachlässigbarem Einfluß auf die Gültigkeit obiger Gleichung.

Unter diesen Voraussetzungen wird man die Ergebnisse der Abb. 2 als ein Maß für die Ionenkonzentrationen im Plasma ansehen können.

Diese Arbeit wurde mit Mitteln durchgeführt, die vom European $\mathrm{Office} A$ ir Research and Development Command zur Verfügung gestellt wurden.

Herrn Prof. Dr. W. Grotн bin ich für die Förderung dieser Arbeit zu großem Dank verpflichtet.

16 A. V. Phelps u. U.S. C. Brown, Phys. Rev. 86, 102 [1952].

17 E. SPenke, Z. Phys. 127, 221 [1950].

\title{
Energieübertragung bei der Fluoreszenz organischer Lösungen
}

\author{
Von Horst Knau \\ Aus dem Physikalischen Institut der Universität Gießen \\ (Z. Naturforschg. 12 a, 881-886 [1957]; eingegangen am 25. Juli 1957)
}

\begin{abstract}
Ein Phasenfluorometer zur Messung von Fluoreszenzabklingzeiten organischer Substanzen bei Elektronenanregung wird beschrieben.

Durch Messung der Abklingzeit von Anthracen und Tetracen in verschiedenen Lösungsmitteln in Abhängigkeit von der Konzentration kann auf Mechanismus und Zeitdauer des Übertragungsprozesses geschlossen werden. Es ergab sich bei großen Konzentrationen eine direkte, strahlungslose Energieübertragung durch Resonanz zwischen einem angeregten Lösungsmittelmolekül und einem Gastmolekül. Bei niedrigen Konzentrationen erfolgt zunächst eine schrittweise Wanderung von Lösungsmittelmolekül zu Lösungsmittelmolekül, bis die Energie einem Gastmolekül so nahe gekommen ist, daß sie direkt zu diesem übergehen kann.

Das Resultat wird bestätigt durch Messungen an Anthracenlösungen, deren Fluoreszenz durch Nitrobenzol gelöscht wurde. Die Diskussion wird dadurch erleichtert, daß diese Messungen sowohl bei UV-als auch bei Elektronenstoßanregung durchgeführt werden konnten.
\end{abstract}

Die stürmische Entwicklung der Kernphysik in den letzten Jahren brachte auch erhöhte Forderungen an die Leistung von Szintillationszählern mit sich. Man sah sich daher veranlaßt, Szintillatoren großer Quantenausbeute und kurzer Nachleuchtdauer zu schaffen. Einige organische Kristalle zeigen diesbezüglich recht gute Eigenschaften. Für manche Zwecke ist man aber gezwungen „feste“ oder „flüssige" Lösungen $\mathrm{zu}$ verwenden.

Wird eine solche organische Lösung mit kurzwelligem UV-Licht, $\gamma$ - oder Korpuskularstrahlung angeregt, so kann eine Übertragung der Anregungsenergie vom Lösungsmittel zum gelösten Stoff erfolgen. Dieser Vorgang ist durch Messung der Spektren und der Quantenausbeute schon vor längerer Zeit bewiesen worden. Messungen der Abklingdauer solcher
Lösungen ermöglichen es nun, auch über den $M e$ chanismus der Energieübertragung sowie über dessen Geschwindigkeit einige Aufschlüsse zu erlangen. Dies war allerdings erst durch die Entwicklung der Meßtechnik in den letzten Jahren möglich, da es sich bei diesen Lösungen um Abklingzeiten der Größenordnung $10^{-10}$ bis $10^{-8}$ sec handelt.

\section{Apparatur}

Der Anregungsteil des Fluorometers besteht aus einem auf ca. $10^{-3}$ Torr evakuierten Glasrohr, in dem eine Haarnadelkathode aus thoriertem Wolframdraht mit etwa 2 Ampere geheizt wird. Die emittierten Elektronen werden durch eine Hochspannung von 30 bis $50 \mathrm{kV}$ zur Anode beschleunigt, die auf Erdpotential liegt. Sie ist durchlöchert und mit einer $10 \mu$-Aluminium- 